\title{
GONDENSED SOLUTIONS OF THE RARE GASES
}

\author{
R. Heastie \\ Department of Physics, Queen Mary College, London, E.1, U.K.
}

The condensed rare gases have a number of properties which make them suitable substances for an experimental test of solution theories. Their molecules have spherical symmetry and have no internal motions and their "radii" may differ by as little as 7 per cent. With the exception of helium, the rare gases crystallize in the same form-face-centred cubic.

Measurements of the total vapour pressure of solid solutions of the two systems $\mathrm{A}-\mathrm{Kr}$ and $\mathrm{Kr}-\mathrm{Xe}$ have been reported ${ }^{1-4}$. These measurements have been compared with theory and in particular with the average potential model theory developed by Prigogine and his associates. This theory leads to an expression for the excess molar free energy $\left(G_{\mathrm{E}}\right)$ of the form:

$$
G_{\mathrm{E}}=\sigma x_{1} x_{2}
$$

where $x_{1}$ and $x_{2}$ are mole fractions of the components and $\sigma$ is a function of temperature (but not of composition) which may be calculated from a number of properties of one component. When $\sigma$ is positive, as it is for the two systems considered here, there exists a critical temperature $T_{c}$, below which the components are insoluble for some values of the composition. The value of the critical temperature, $T_{c}$, is given by $\mathcal{T}=(1 / 2 R) \sigma$. From the measured vapour pressure, $P$, at several temperatures, $T, \sigma$ may be determined as a function of $\mathcal{T}$. The temperature $T_{\mathrm{c}}$ at which $\sigma=2 R T$ may thus be estimated. The values of $T_{c}$ so calculated ${ }^{1,2}$ are shown in Table 1. No direct experimental evidence of the existence of two solid phases has been obtained for either the $\mathrm{A}-\mathrm{Kr}$ or the $\mathrm{Kr}-\mathrm{Xe}$ system. Some recent experiments with the latter system in which indications of a phase separation have been observed, are described below.

In the investigations of $\mathrm{A}-\mathrm{Kr}$ solutions by Walling and Halsey ${ }^{2}$ and $\mathrm{Kr}-\mathrm{Xe}$ solutions by Freeman and Halsey ${ }^{1}$ an amount of the heavier gas was condensed into a thermostated vessel at a temperature $T_{1}$. Subsequently, amounts of the other component were condensed into the same vessel. The solid mixtures were allowed twenty-four hours to attain equilibrium before the vapour pressure was measured. From these measurements a mean value of $\sigma, \bar{\sigma}$ for solutions of different compositions was calculated for each value of $T_{1}$. These values of $\bar{\sigma}$ are compared with the prediction of the average potential theory in Table 1 . Considering the nature of the theory, the agreement is good.

In the author's experiments the two gases were mixed in the gaseous state and condensed into a calorimeter which was subsequently heated. Vapour pressure measurements were made at intervals during the heating 


\section{R. HEASTIE}

period $(6-10 \mathrm{~h})$. The measurements were extended through the heterogeneous region until the mixtures were liquid. By this means the solidus and liquidus curves were determined. In the case of $\mathrm{A}-\mathrm{Kr}$ mixtures the measurements were found to be inconsistent with equation (1), i.e. $\sigma$ as calculated by this equation was a function of composition. Nevertheless, the actual magnitude of the vapour pressure predicted by the average potential theory is in very good agreement with the observed vapour pressure $^{3,4}$.

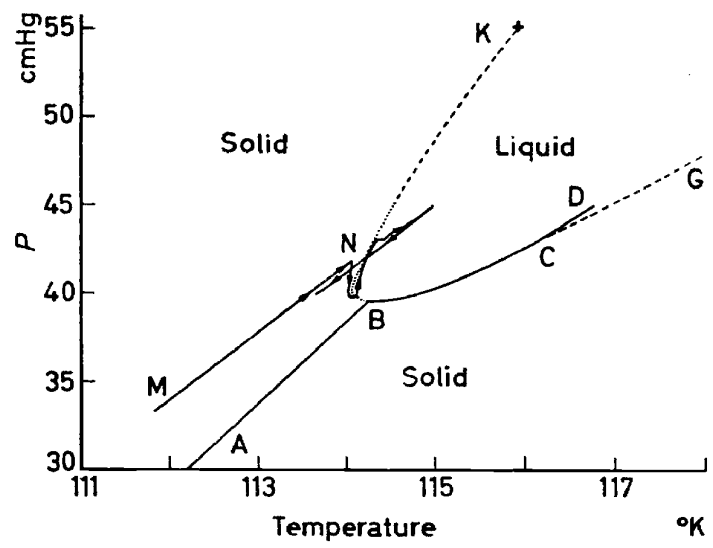

Figure 1. The $P-T$ diagram for $\mathrm{Kr}-\mathrm{Xe}$ mixtures: $\mathrm{K}$, triple point of xenon, $\mathrm{KBCG}$ threephase curve, $\mathrm{ABCD}$ and $\mathrm{MN}$, curves for mixtures for which $x=0.28$ and $x=0 \cdot 11$ respectively

The $\mathrm{Kr}-\mathrm{Xe}$ system has been examined by the same method in the range $x=0$ to $x=0.44$ where $x$ is the mole fraction of xenon. In Figure 1 , the $P-T$ graph ABCD of a mixture of composition $x=0.28$ is shown $(P=$ the total vapour pressure at the temperature $T)$. The phases present in the mixture are: along $\mathrm{AB}$ solid and vapour, along $\mathrm{BG}$ solid, liquid and vapour and along $\mathrm{CD}$ liquid and vapour. The three-phase portions ( $\mathrm{BC}$, in this case), for all mixtures lie on the continuous curve KBCG. The liquid-solid phase transition points from which the phase equilibrium diagram, Figure 2, is drawn may be discovered from the intersection of the curves $\mathrm{AB}$ and $\mathrm{DC}$ with the three-phase curve.

Within the ranges $0<x<0.04$ and $0.27<x<0.44$ the $P-T$ graphs were of the form ABCD, i.e. the phase transition points and the three-phase curve were well defined. In the intermediate range $0.04<x<0.27$ irregularities in the $P-T$ curves make the exact form of the solidus and liquidus and three-phase curve (shown dotted in this region in Figure 1) uncertain. It is quite possible that a eutectic point exists, in which case the three-phase curve would have a cusp at $P \bumpeq 40 \mathrm{~cm} \mathrm{Hg}$ and $T \bumpeq 114^{\circ} \mathrm{K}$.

The uncertainty in both Figures $I$ and 2 arises from irregularities in the results obtained for all mixtures in this range, as exemplified by the curve MN for a mixture $x=0 \cdot 11$. As this mixture was heated a sudden fall in $P$, accompanied by an absorption of heat, was observed. After the mixture had melted completely the calorimeter was cooled and the subsequent behaviour of the mixture may be seen from Figure 1. (The behaviour of 


\section{CONDENSED SOLUTIONS OF THE RARE GASES}

these mixtures on cooling is not properly understood and is now the subject of further investigation.) Many mixtures within this intermediate range have been examined and all the $P-T$ curves take the same form as $M N$ on Figure 1. The maximum absorption of heat recorded is approximately $90 \mathrm{~J} /$ mole. It may be seen from Figure 2 that the measurements of Freeman and Halsey do not cover this region of the phase equilibrium diagram.

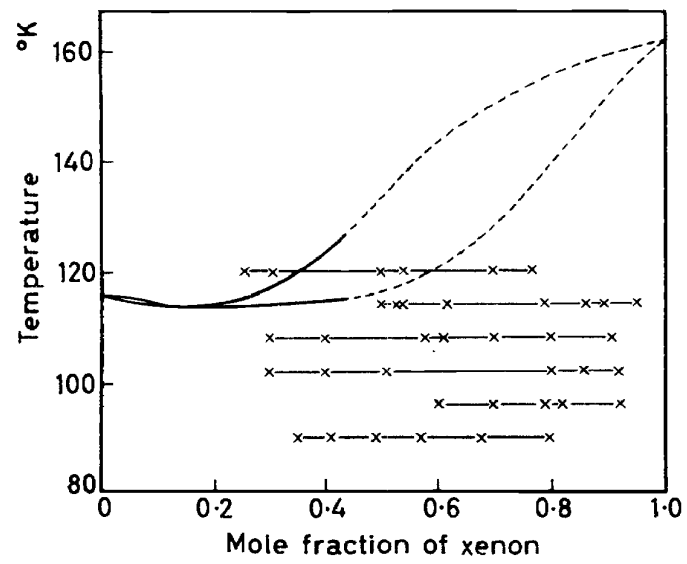

Figure 2. The solid-liquid phase equilibrium diagram of the $\mathrm{Kr}-\mathrm{Xe}$ system. Measurements of vapour pressure were made at points marked by crosses by Freeman and Halsey ${ }^{1}$

The behaviour of the mixtures within this limited range of composition is consistent with the assumption that the gases, on condensation, form a two-phase solid mixture, the pressure fall and absorption of heat being associated with a transition to one single solid phase. To test whether an inhomogeneous mixture was created solely by the method of condensation used, the condensation temperature was varied between $65^{\circ} \mathrm{K}$ and $110^{\circ} \mathrm{K}$. In all cases the measured $P-T$ curves took the same form. Since, however, the solid mixtures are clearly not in a state of thermodynamic equilibrium, it is not possible to assert that the existence of a stable two-phase region in the phase equilibrium diagram of the $\mathrm{Kr}-\mathrm{Xe}$ system has been demonstrated.

Table 1. Solid solution data compared with theory

\begin{tabular}{l|c|c|c|c|c}
\hline \multirow{2}{*}{ System } & \multicolumn{2}{|c|}{$\bar{\sigma}(\mathrm{J} /$ mole $)$} & $\begin{array}{c}\text { Temp. } \\
\left({ }^{\circ} \mathrm{K}\right)\end{array}$ & Ref. & $\begin{array}{c}T_{\text {c estim. }} \\
\left.{ }^{\circ} \mathrm{K}\right)\end{array}$ \\
\hline \begin{tabular}{c|c|c|c|c|} 
Exp. \\
Argon-krypton
\end{tabular} & $\begin{array}{c}\text { Calc. } \\
750\end{array}$ & 685 & 83 & 2 & $56 \pm 8$ \\
\hline Krypton-xenon & 1350 & 2320 & 105 & 1 & $91 \pm 2$ \\
\hline
\end{tabular}

\section{References}

${ }^{1}$ M. F. Freeman and G. D. Halsey. F. Phys. Chem., 60, 1119 (1956)

${ }^{2}$ J. F. Walling and G. D. Halsey. F. Phys. Chem., 62, 752 (1958)

3 R. Heastie and G. O. Jones. Nuovo cimento Suppl., 9, 365 (1958)

${ }^{4}$ R. Heastie. Proc. Phys. Soc. (London), 73, 490 (1959) 